Fanum

Sociológico

\section{Forum Sociológico}

Série II

$29 \mid 2016$

Número 29

\title{
Vejez y sociedad en México: Las visiones construidas desde las Ciencias Sociales
}

Velhice e sociedade no México: As visões construídas a partir das Ciências Sociais old age and society in Mexico: The visions built from the Social Sciences

\section{Fernando Bruno y Jesús Acevedo Alemán}

\section{OpenEdition}

\section{Journals}

\section{Edición electrónica}

URL: https://journals.openedition.org/sociologico/1453

DOI: 10.4000/sociologico. 1453

ISSN: 2182-7427

Editor

CICS.NOVA - Centro Interdisciplinar de Ciências Sociais da Universidade Nova de Lisboa

Referencia electrónica

Fernando Bruno y Jesús Acevedo Alemán, «Vejez y sociedad en México: Las visiones construidas desde las Ciencias Sociales», Forum Sociológico [En línea], 29 | 2016, Puesto en línea el 31 diciembre 2016, consultado el 29 marzo 2022. URL: http://journals.openedition.org/sociologico/1453 ; DOI: https://doi.org/10.4000/sociologico.1453 


\title{
VEJEZ Y SOCIEDAD EN MÉXICO: LAS VISIONES CONSTRUIDAS DESDE LAS CIENCIAS SOCIALES VELHICE E SOCIEDADE NO MÉXICO: AS VISÕES CONSTRUIDAS A PARTIR DAS CIÊNCIAS SOCIAIS \\ OLD AGE AND SOCIETY IN MEXICO: THE VISIONS BUILT FROM THE SOCIAL SCIENCES
}

\author{
Fernando Bruno \\ - Universidad Autónoma de Coahuila, Facultad de Trabajo Social, Cuerpo Académico de Grupos Vulnerables \\ Jesús Acevedo Alemán \\ Universidad Autónoma de Coahuila, Facultad de Trabajo social, Cuerpo Académico de Grupos Vulnerables
}

\begin{abstract}
Resumen
La investigación social de la vejez en México, ha mostrado significativos avances en los últimos años, con la particularidad de abordarse desde enfoques diversos que se iniciaron en la Geriatría hasta llegar a la Sociología, Antropología y el Trabajo Social. Estos derroteros permitieron la construcción de visiones y teorías sobre la vejez, evaluando los impactos de este fenómeno sobre la sociedad. Este artículo tiene como objetivo analizar la construcción social de la vejez y el envejecimiento en México desde las Ciencias Sociales y brindar un aporte sobre los desafíos futuros ya que, el proceso de envejecimiento es cada vez más notable en el país, así como las deficiencias institucionales y culturales para integrar socialmente a la población de la tercera edad. Entre los hallazgos se destaca que hasta el momento no se ha podido incorporar hacia las políticas sociales, provocado por una visión a corto plazo de los mecanismos para mejorar la situación de las personas mayores de 60 años.
\end{abstract}

Palabras-clave: envejecimiento en México, edad, desafíos de la vejez, problemáticas de la vejez

\section{Resumo}

A investigação social sobre o envelhecimento no México tem mostrado progressos significativos nos últimos anos, com a particularidade de partir de diversas abordagens de Geriatria e alcançar a Sociologia, Antropologia e Serviço Social. Estes caminhos levaram à construção de visões e teorias sobre o envelhecimento e à avaliação do impacto do fenómeno na sociedade.

Este artigo visa analisar a construção social da velhice e do envelhecimento no México a partir das Ciências Sociais. Contribui para a identificação de futuros desafios, uma vez que o processo de envelhecimento é cada vez mais perceptível no país, bem como de deficiências institucionais e culturais para integrar socialmente as pessoas da terceira idade. Entre os resultados, salienta-se que, até agora, não tem sido possível incorporar o sentido das políticas sociais, devido a uma visão de curto prazo dos mecanismos criados para melhorar a situação das pessoas idosas.

Palavras-chave: envelhecimento no México, idade, desafios de idade, os problemas da velhice

\section{Abstract}

Social research on aging in Mexico, has shown significant progress in recent years, with the particularity of addressed from various approaches that were started in the Geriatrics until reach the Sociology, Anthropology and the Social Work. These paths led to the construction of visions and theories on aging, evaluating the impact of this phenomenon on society. 


\begin{abstract}
This article aims to analyze the social construction of old age and aging in Mexico from the Social Sciences and provide a contribution on the future challenges since, the aging process is increasingly noticeable in the country, as well as the institutional weaknesses and cultural to integrate socially to the people of the old age. Among the results it is stressed that so far has not been able to incorporate toward social policies, caused by a short-term vision of the mechanisms for improving the situation of older persons.
\end{abstract}

Keywords: aging in Mexico, age, challenges of aging, problems of aging

\section{Introducción}

Reflexionar acerca de la edad y los roles que le otorga la sociedad a cada una de ellas requiere romper con múltiples prenociones, porque tanto la niñez, la juventud y la vejez están cargadas de estereotipos que se construyen socialmente e impiden alcanzar una visión más precisa del tema.

Sin embargo, no todos los grupos presentan el mismo problema, por el contrario, existe una mayor claridad en cuanto al rol que deben desempeñar los niños - son dependientes en todos los sentidos y se necesita socializarlos - y los jóvenes, aunque empíricamente se demuestre que una gran cantidad de éstos no puedan jugar el rol social deseado. Los viejos, traen bajo ese rótulo una complejidad mayor producto de la ambigüedad de su posición en la sociedad, además de sostener una carga negativa sobre su situación, que no sucede necesariamente con otras categorías de edad. Se expresa esto con respecto a los roles, que no significa de ningún modo que sea simple la investigación sobre la niñez o la juventud. Por el contrario, los procesos de investigación para cualquier grupo de edad presentan desafíos intelectuales complejos.

La principal razón de esta visión deriva en una serie de hechos que tienden a dibujar la imagen de la vejez como deterioro, dependencia, en breve como una carga para todos. Esta percepción deriva de la construcción social de la vejez, que desde las Ciencias Sociales y los diferentes abordajes se fueron erigiendo en consonancia con los cambios sociales. Este artículo se construye a partir de la compleja relación entre sociedad y vejez, con los intentos de explicación y las visiones presentes. La riqueza heurística del tema y el debate generado, ha dejado aún espacios por indagar y otros por retomar.

Por otra parte, realizar este tipo de ejercicio desde México tiene implicaciones particulares porque entre los roles, las normas y las costumbres locales, se suma el tiempo que también ha transformado las características y magnitudes de la vejez. En esta dinámica se inscriben respuestas académicas que exponemos a partir del siguiente trabajo analítico que comienza en un primer momento por efectuar un recorrido sobre las principales problemáticas ligadas a la vejez y las investigaciones que le han dado respuesta. Posteriormente realizaremos una síntesis crítica, resaltando la construcción social de la vejez en México, a partir de algunos de los hallazgos, con el objetivo final de reelaborar una nueva agenda de los desafíos para afrontar nuevas investigaciones.

Para tales propósitos, el escrito siguió una argumentación articulada a partir de las temáticas más sobresalientes sobre las características de los adultos mayores y sumando el proceso de envejecimiento en México, considerando para ello, los autores que más aportes han realizado en cada una de las temáticas, seleccionando las fuentes de datos bibliográficas en base a la calidad de los aportes teóricos. La selección de las temáticas responde a los desafíos que el conocimiento sobre la vejez, necesita afrontarse México.

Finalmente, la metodología de exposición utilizada fue cronológica, en el sentido de exponer en cada una de las temáticas los aportes que históricamente se desarrollaron primero y su evolución. En resumen, se está frente a un trabajo de revisión de investigaciones, para lograr una síntesis crítica del conocimiento existente actual sobre las situaciones que caracterizan a los adultos mayores en México, en diferentes áreas.

\section{Las investigaciones sobre la vejez: la complejidad inicial de la edad social}

Al comenzar la investigación sobre el tema de la vejez en México, existen algunos hechos que a priori se enfatizan, como por ejemplo la velocidad del proceso de envejecimiento en México y los desafíos que ello implica en el plano político, social y económico. Además, la mayor esperanza de vida para hombres y mujeres más que un logro positivo, parece transformarse en una franca carga social negativa. De la misma manera, se destaca otro aspecto que es el del "bono demográfico" también como una de las preocupaciones principales en torno a la relación del mercado de trabajo y los adultos mayores. El bono demográfico es una ventana de oportunidad que existe en México en las primeras décadas del presente siglo, en donde por cada persona dependiente habrán dos personas activas. Esta situación se extenderá hasta la primera mitad 
del siglo XXI cuando el mismo indicador comenzará a revertirse.

Ahora bien, lo que se ha mencionado se relaciona con un proceso demográfico que es complejo y multicausal, pero de los viejos, como individuos con sus características, significa un abordaje diferente porque se refiere a una categoría social.

Es fundamental por tal motivo diferenciar conceptualmente envejecimiento y vejez, puesto que existe entre ellos una conexión muy estrecha porque son parte constitutiva de la cuestión de la edad. La vejez es considerada una etapa en la vida de los sujetos que conlleva implícito roles sociales específicos, aunque no se trata de una clasificación aceptada de manera unívoca; el envejecimiento en cambio es visto como un proceso que comienza desde el nacimiento del ser humano y tiene que ver con el cumplimiento de los años. Otra definición es aquella que menciona que el envejecimiento es un proceso universal, continuo y progresivo, que lleva finalmente al deterioro y la muerte, esto desde una concepción eminentemente biológica (Serrani, 2012).

Otra complejidad existe también alrededor de la definición de vejez y los sinónimos que se utilizan - edad avanzada, anciano, adulto mayor - provocado por la falta de acuerdo sobre los límites de la edad para ingresar a la categoría de la vejez, dado que para algunos autores el ingreso son los 60 y para otros los 65 años de edad. Pero hay un punto de acuerdo y es que con la vejez, es decir con la acumulación de tiempo en el ser humano, aparecen cambios físicos que son acompañados también por cambios en las relaciones sociales.

Esto significa que la sumatoria de edad trae consigo transformaciones personales, en las condiciones físicas, en la independencia económica y funcional, como también se asiste a la modificación de roles dentro de la familia y en la sociedad, la participación en el mercado laboral, además del uso del tiempo (Aranibar, 2001).

De la misma manera que el envejecimiento adquiere rasgos que son propios a cada país, la vejez también tiene características que le son inherentes a las condiciones sociales y económicas imperantes. Si bien no existe dificultad en calcular la edad cronológica (una persona cumple años una vez cada 365 días), "ser viejo" o "adulto mayor", es más complejo el pasaje de la definición a la operacionalización porque se define y redefine en el tiempo variando de una cultura a otra. A raíz de esta complejidad, se han desarrollado numerosas investigaciones sobre la condición de los viejos.

Por ello el envejecimiento se ha convertido en una cuestión de interés para la política pública de orden mundial, debido a las proporciones por país de personas de 60 años o más, que siguen aumentando de manera absoluta y porcentual en detrimento de las edades adultas y jóvenes. Por ejemplo, indica la OMS (2015) que en 2012 las proyecciones para 2050, solo Japón tiene una proporción superior al $30 \%$ de adultos mayores. Sin embargo, en la segunda mitad del siglo, muchos países tendrán una proporción similar, se trata de países de Europa y América del Norte, pero también de Chile, China, la Federación de Rusia, la República de Corea, la República Islámica de Irán, Tailandia y Vietnam.

\section{Las problemáticas de la vejez y sus respuestas en México}

Particularmente en México, el envejecimiento y la atención a sus necesidades es un fenómeno que encierra una compleja problemática, que según Acevedo, Bruno, Trujillo y López (2015), el país, como sus políticas sociales están estructurados para responder a las demandas de una población joven. El envejecimiento de la población implicará modificar el gasto social, reduciendo por ejemplo la construcción de escuelas y el número de maestros, aumentando los servicios destinados a personas mayores; reduciendo los espacios pediátricos en los hospitales, aumentando de tal manera los geriátricos; requiriéndose por consecuencia profesionales del cuidado y atención a las necesidades de dicho sector, dando así pasos hacia un envejecimiento saludable (OMS, 2015).

Destacando los propios autores que la situación que guardan los adultos mayores en México es heterogénea, en donde no se puede afirmar que todos envejecen bajo las mismas condiciones o que gozan de los mismos privilegios, de igual manera tampoco se puede negar que la población del país está avanzando en edad, y que en conjunto con la sociedad necesitan adaptarse a esta nueva situación, y hacer los cambios necesarios para atender las necesidades y demandas de esta población en crecimiento (Acevedo et al., 2015).

Ahora bien, en el país, durante las últimas cuatro décadas experimentó un acelerado cambio en los ámbitos demográfico y epidemiológico, lo que consolidó su paso a una etapa avanzada de la transición demográfica (Sánchez, 2008). Tales tendencias, seguidas por los factores del cambio demográfico, determinaron no sólo el crecimiento de la población, sino también marcaron cambios en su composición por edades. Por ejemplo, en 1950, había un millón 400 mil adultos mayores; en el año 2000, la población de 65 años o más representaba 4.6\% (4.6 millones de personas) de la población total; en 2005, 8.3 millones de personas tenían 60 años de edad y más de los cuales el $46.69 \%$ representaron hombres y el $53.31 \%$ mujeres.

En tal sentido, para el año 2010, 10 millones de mexicanos se situaron en más de 60 años, con un marcado aumento de las tasas de fecundidad y la proporción de jóvenes irá disminuyendo paula- 
tinamente mientras que la población mayor de 60 años aumentó su volumen hasta representar $11.7 \%$ de la población total (Gutiérrez, Serralde y Guevara, 2007; Parra y Quintero, 2007).

Por su parte la (Zúñiga y Vega, 2004) estiman que para el año 2050 , de acuerdo con la proyección poblacional, uno de cada cuatro mexicanos será mayor de 60 años de edad, calculando que actualmente $7 \%$ del total de la población es mayor de 60 años. Datos que problematizan el envejecimiento por el cual está atravesando México, cuyas implicaciones no sólo repercutirán en este grupo de edad, sino también para las familias, los cuidadores, las instituciones y la sociedad en su conjunto.

\section{Esbozando una problemática de interés}

Ahora bien, además de los intereses relacionados con la demografía y el proceso de envejecimiento en México, hubieron investigaciones que se enfocaron a temáticas que tienen más que ver con las características de los viejos y las dinámicas sociales y económicas que ellos impulsan (Ham, 2003; INEGI, 1990; Montes de Oca, 1999; 2010; OMS, 2015; Partida, 2005; Reyes, 2006; Tuirán, 1999; Zúñiga y Vega, 2004).

Pero antes de destacar las temáticas sobre los adultos mayores, conviene dejar en claro un punto importante. Si el envejecimiento de la población, como ya se vio, afecta a todas las sociedades y por eso se trata de un proceso homogéneo, los adultos mayores como sujetos de estudio, como categoría son portadores de una gran heterogeneidad, cuestión que ha fundamentado justamente la presencia de una gran cantidad de investigaciones sobre el tema.

La heterogeneidad de los viejos es la consecuencia del lugar que ocupa en la sociedad el sujeto, dado que se caracteriza a variar a lo largo del tiempo en consonancia a la estructura de oportunidades movilidad social - que son diferenciales para hombres y mujeres, para pobres y ricos o que se trate de indígenas, blancos o mestizos (Montes de Oca, 2010).

Cuando nos referirnos a la vejez de manera general, es decir como una etapa dentro de un continuo temporal del ciclo de vida de una persona, nos percatamos que no todos los individuos envejecen de la misma manera. Dentro de esta población se encuentra toda una gama de paisajes diferentes, cristalizando las particularidades de cada trayectoria de vida.

Como se mencionó en el inicio de este trabajo, las investigaciones sobre la vejez y el envejecimiento no datan de hace muchos años en México, uno de los primeros trabajos fue La tercera edad en México, 1993. IX Censo General de Población y Vivienda (INEGI, 1990). Por el contrario, es a partir de la década de 1990, que comienzan a aparecer la mayoría de los aportes. Es sobre esta situación, además del propio interés de este trabajo, que es posible agrupar lo que en la actualidad se sabe sobre los adultos mayores en México, alrededor de algunas temáticas principales.

En primer lugar, se pueden situar los fundamentos que han construido la vejez como fenómeno de estudio en las Ciencias Sociales.

Actualmente no quedan dudas que no hay un solo enfoque o disciplina que investigue la vejez. El proceso de envejecimiento y el interés que han despertado las personas de la tercera edad confluyeron hacia la aparición de una vasta bibliografía. Sin embargo, la Gerontología y las Ciencias Médicas fueron inicialmente las primeras en abordar el tema de la vejez, aunque la impronta estaba caracterizada por una el rótulo de problema y patología, más que como un fenómeno social en construcción.

La preocupación inicial es el aislamiento social de las personas mayores y su baja autoestima. En esta tónica las nociones utilizadas rotulan a la vejez como una locura, o degradación, muy en sintonía con los primeros avances de la geriatría.

La Gerontología es principalmente la disciplina que se ocupa del estudio o conocimiento de los más viejos, pero como señala Osorio (2006), muchos estudios sociales y gerontológicos respoden a una demanda de qué hacer con el envejecimiento dimensión empírica y aplicada -, dejando de lado todo aspecto teórico que no ha sido desarrollado hasta nuestros días (Osorio, 2006).

De esta manera la Gerontología, como menciona Johnson (2001), ha estado dominada por el aspecto empírico y patológico del envejecimiento, más preocupada por la cantidad de datos, que no han sido acompañada de un cuerpo teórico. Podríamos decir que la Gerontología se acerca más a la Medicina que a la Sociología en la manera de tratar inicialmente el tema.

Justamente las críticas iniciales a la Gerontología giraban en torno a la falta de explicaciones teóricas para abordar la diversidad de experiencias de las personas de edad en la sociedad. Es decir, existía una concentración en el problema funcional de la población envejecida, como puede ser la preocupación por la falta de autonomía.

Esta crítica esboza a partir de la historia de los inicios del tema de la vejez en los años 70, donde la Biología, la Medicina y la Psicología habían hecho los principales aportes. Producto de una reconceptualización, en la actualidad la Gerontología no estudia solamente la edad, sino el envejecimiento y el proceso de envejecer. Para los autores es el estudio de la vejez, teniendo en cuenta fenómenos biológicos, psicológicos y sociales lo que caracteriza actualmente la Gerontología (Scolich, 2005).

En síntesis, hay que mencionar que la gerontología tradicional de asendencia positivista y biomédica fue, con el avance de la teoría social 
contemporánea, reemplazado por la gerontología social crítica, reclamando con éxito el exámen crítico de las construcciones conceptuales con un fuerte contenido feminista (Yuni y Urbano, 2008).

Además, se han elaborado otras teorías que han ido ocupado el vacío explicativo en torno a la vejez. Acompañando al despliegue teórico originado a partir de los trabajos de Talcott Parsons, principalmente en la década de los sesenta, se encuentra la teoría de la desvinculación creada por Cummings y Henry en los sesenta, "que centró su interés en las personas de edad en cuanto individuos que ya no participaban en el mercado laboral y por lo tanto, ya no formaban parte de una esfera esencial de la sociedad" (Osorio, 2006: 7). Cuarenta años más tarde, encontramos posturas y enfoques hacia la vejez "activa" o "exitosa", que en muchos casos se centran en una actitud hacia la vida, más que en una teoría.

Para Johnson (2001), como especielista en salud y política social, el razonamiento de los investigadores interesados en el envejecimiento debería girar en torno a tres ejes principales. En primer lugar, sobre la población considerada mayor por su longevidad y esperanza de vida. Desde hace algunas décadas una gran cantidad de estudios gerontológicos han insistido sobre las dificultades funcionales de los viejos tomando como ejemplo la salud y la incapacidad de desarrollar su autonomía (Johnson, 2001).

El segundo eje presenta el tema del envejecimiento desde el punto de vista evolutivo y el énfasis es puesto en cómo una especie se desarrolla y envejece, sin olvidar las cuestiones biológicas, psicológicas y sociales que se interrelacionan. Para el autor, la mayoría de las investigaciones sobre este tema se realizan de forma transversal, y el enfoque longitudinal es necesario para analizar el envejecimiento en términos de proceso.

El tercer y último eje, consiste en una aproximación hacia la vejez como un aspecto de la estructura y de comportamiento de las especies. Particularmente este eje ha capturado la atención de sociólogos, además de otros científicos, dado que se examina las poblaciones humanas, así como las organizaciones sociales que se crean y modifican como respuesta a esquemas de socialización, de acceso a la edad adulta y la jubilación. En este punto, las explicaciones necesarias son aquellas que relacionan la edad con las instituciones y la sociedad, como por ejemplo el mercado de trabajo, la salud y el sistema de pensiones (Johnson, 2001).

Existen algunos puntos de encuentro entre el anterior autor y el sociólogo español Sánchez (2000), para quien en los estudios socioeconómicos sobre los adultos mayores se abordan las siguientes temáticas con sus críticas:

1. La dominación de una perspectiva ideológica (sin negar que hayan varias en juego).
2. La hegemonía de lo "económico" sobre lo "sociológico", y de lo "social" sobre lo "sociológico".

3. La preeminencia de lo macro sobre lo micro.

4. La diversidad y dispersión de los objetos de estudio.

5. La falta de definición y de clarificación conceptual sobre los efectos benefactores de las conductas y los comportamientos del mayor sobre la economía.

6. La servidumbre de otros estudios que incluyen "lo económico" directa o tangencialmente como una variable discreta, y que están referidos al comportamiento de diferentes grupos etarios, en donde suele incluirse al grupo de mayores de 65 años.

7. La falta de articulación y posiblemente de permeabilidad en este campo, de la Sociología de la Vejez con otras Sociologías: Sociología de la Salud, de la Familia, de la Población, Industrial y del Trabajo, del Género, de la Vida Cotidiana, del Consumo, del Ocio.

8. La dependencia de "lo social", de "lo económico"; y la dominancia de la perspectiva del bienestar social y de la política social (estudios sobre pobreza) sobre los estudios básicos sobre comportamiento y valores económicos del mayor (Sánchez, 2000: 41).

Aunque el autor escribe desde otro contexto mundial, los puntos no parecen estar alejados de las necesidades que desde México se imponen en la agenda de investigación sobre la vejez y el envejecimiento de la población. La dependencia de lo económico, como se señala, cristaliza la preocupación de la opinión pública sobre la vejez vista exclusivamente como un problema, que va más allá de la construcción de una problemática social compleja y dinámica.

En otra área temática, los conceptos de pobreza y vulnerabilidad en la vejez, han estado presentes desde el inicio en las investigaciones. Este tema cobra una relevancia mayor en México, un país que actualmente no ha podido resolver los desafíos impuestos por una sociedad cada vez más desigual y con un alto grado de rezago social. Sobre este aspecto, para mencionarlo de manera breve, se ha mostrado que el envejecimiento de la población en México se lleva a cabo en un contexto caracterizado por un alto índice de pobreza, una amplia desigualdad social y económica producto de una redistribución regresiva del ingreso, y en el plano del mercado de trabajo, con una clara deficiencia en la creación de empleos de calidad y regulados (Zúñiga y Vega, 2004).

Es por ello que las características de la sociedad mexicana son poco favorables para el panorama del envejecimiento demográfico ya que existe una 
correlación entre ancianidad y empobrecimiento, como resultado de la falta de ahorro o acumulación de bienes durante la trayectoria de vida de una persona (Ribeiro y Mancinas, 2009). En esta misma dinámica, Zúñiga y Gomes (2002), señalan que la menor incidencia de la pobreza en México se encuentra a partir de los 45 años de edad, pero, a partir de los 65 años, momento del cese institucional de actividades laborales, comienza a ascender el impacto de la pobreza hasta alcanzar un $31.1 \%$ en el grupo de 75 años y más de edad.

Para Woolf (1989, citado por Huenchuan y Guzmán, 2003), la pobreza en los adultos mayores está relacionada con la vulnerabilidad en el ciclo de vida, de esta manera la edad, pasa a conformar una condición de fragilidad que permite el descenso brusco de una situación de subsistencia, hacia la pobreza con mayor facilidad que en otras etapas de la vida. Si la vejez se asocia con pobreza es porque está presente el proceso de retiro de la actividad laboral y la muerte de la pareja en muchos casos, que reducen sensiblemente los recursos económicos y aumenta así las probabilidades de caer en la falta de recursos (Huenchuan y Guzmán, 2003).

Asimismo el CONAPO (2003) construye índices de desarrollo social para seis etapas de la vida, y para ello tiene en cuenta dimensiones como la salud, educación entre otras. Los resultados de este trabajo muestran que los mayores de 60 años de edad en México exhiben las condiciones de desarrollo social más precarias. Una de las dimensiones construidas para esta etapa fue la capacidad de gozar un nivel de vida adecuado y los resultados más desalentadores nuevamente se encuentran en edades avanzadas (CONAPO, 2003).

La situación es que se asocia el riesgo del empobrecimiento a la reducción de oportunidades laborales para las edades avanzadas, además de la "pérdida paulatina de sus capacidades físicas y de salud, como también la a insuficiente cobertura de los sistemas de seguridad social y la dependencia de las transferencias familiares" (Montes, 2009: 23).

En este escenario, es normal encontrar miradas pesimistas con respecto al envejecimiento de la población en México; aun así es fundamental recalcar que "la calidad de vida de las futuras generaciones de adultos mayores dependerá en buena medida de la capacidad de la sociedad para construir y consolidar las instituciones encargadas de procurar su protección y bienestar" (CONAPO, 2003: 16). Pero, lo cierto es que el colectivo de ancianos es heterogéneo - no todos son pobres o sufren un proceso de empobrecimiento - y su situación tiene más que ver con las características sociales y las oportunidades acumuladas a lo largo de la vida y el contexto sociohistórico en el que se desenvuelven.

A partir del panorama anterior aparece otra área de investigación y sobre todo una línea de acción con respecto a la situación de los adultos mayores; se trata de las políticas sociales.

El orígen de la Política Social hacia los adultos mayores se encuentra en el "plan de Viena". En efecto, en 1982 se llevó a cabo en la capital europea la primera asamblea mundial sobre envejecimiento y a partir de allí surge el plan mencionado que, de manera resumida, contiene indicaciones y recomendaciones que forman una política universal para enfrentar los problemas ligados a la vejez y el envejecimiento (Viveros, 2001). A partir de estas tesis, los gobiernos no han cesado en incorporar en sus agendas el tema del envejecimiento y cómo enfrentar los desafíos sociales que resultan de este fenómeno. Las respuestas ensayadas han sido variadas de acuerdo al país y los contextos políticos que se viven.

México no es ajeno a esta dinámica y el 25 de julio de 2002, bajo la presidencia de Vicente Fox, se promulga la ley de los derechos de las personas adultas mayores para lo cual se crea el Instituto Nacional de las Personas Adultas Mayores (INAPAM), que es una de las instituciones rectores en la atención de los adultos mayores. La población objetivo son los residentes en cualquier parte del territorio nacional que tengan 60 años o más, mismas que se denominan Personas Adultas Mayores.

A partir de este esquema se puede interpretar que los impulsos internacionales sobre el problema de la vejez, se tradujeron en México por un conjunto de iniciativas que traducen el valor moral y normativo de intentar revertir el rezago sufrido por este grupo etario. El hecho social que va a impulsar las políticas hacia los viejos es la constatación de un envejecimiento que se dará a gran velocidad y los rezagos que hasta hoy en día, presenta este grupo poblacional.

Una de las políticas que ha recibido atención de parte de los investigadores es el programa "70 y más" implementado a partir de mayo de 2004 . El programa tiene como objetivo principal contribuir a abatir el rezago que enfrentan los adultos mayores de 70 años en adelante, mediante políticas orientadas a fomentar su protección social con acciones de promoción, participación social y el otorgamiento de un apoyo económico (SEDESOL, 2010).

La concepción sobre la vejez que predomina las políticas es la que la percibe como carencias económicas, físicas y sociales. Las primeras se expresan en falta de ingresos, las segundas en falta de autonomía, las terceras en falta de roles sociales. Por lo tanto, la intervención se define a partir de lo que las personas mayores no poseen en comparación con otros grupos sociales. Los instrumentos de política son así paliativos para superar esas pérdidas o carencias (Huenchuan, 2011).

A raíz de ello, algunos autores (Bueno y Dávila, 2010; Rodríguez, 1999), pugnan por el diseño de 
políticas hacia los adultos mayores que sean integrales, lo que significa articular los diferentes dispositivos de atención. Esta postura equivale a sostener una mirada diferenciada entre políticas generales y políticas específicas para los integrantes de este grupo de edad. Cada grupo de edad y rango, tiene sus propias necesidades y urgencias, que hay que reconocer para construir políticas adecuadas (Rodríguez, 1999). Por ejemplo, a partir de la vejez las cuestiones ligadas a la salud cobren mayor importancia, así como a partir de los 65 años de edad la seguridad económica es un tema determinante para el bienestar de los viejos.

Recapitulando, el mismo autor señala que lo dicho anteriormente "refuerza la idea de que una política social sobre el envejecimiento y la vejez no puede fincarse sobre una sumatoria de programas existentes y debe, más bien, basarse en una reflexión respecto a las necesidades presentes y sobre todo las futuras de este grupo" (Rodríguez, 1999: 65).

Dentro de este debate, existe un área que ha recibido menor atención, pero que ya ha sido planteada y es el tema de las políticas laborales hacia los adultos mayores. Dadas las proyecciones sobre la estructura de edades de la población, uno de los principales desafíos se encuentra en "diseñar políticas de empleo y acceso al mercado laboral, que permitan la mantención o recuperación de empleos, distinguiendo la vocación y deseos de seguir activos junto a la de imposición de continuar trabajando por necesidades económicas" (Viveros, 2001: 21).

Retomando la necesaria integración de las políticas sociales hacia los adultos mayores, este escrito es también una oportunidad para contribuir al debate sobre este tema. La postura que se puede adoptar es iniciar el recorrido hacia una reflexión sobre los adultos mayores, desde las políticas de empleo y no solamente desde los grupos vulnerables. También se plantea la necesidad de una mirada a largo plazo que incluya un enfoque relativo al mercado de trabajo desde las trayectorias laborales (Pacheco, 2009).

Otra temática que ha recibido un fuerte interés es el tema del envejecimiento y vejez desde el enfoque del género, en donde uno de los trabajos más sólidos desde la Sociología es el de Arber y Ginn (1996). Además de éstas autoras, otras investigaciones pusieron en evidencia la estructura de oportunidades que tiene la población según el sexo en esferas sociales tales como la educación, el trabajo, la salud y la seguridad social a lo largo de toda la vida (Montes de Oca, 2010). La conexión entonces entre género y envejecimiento devela la distribución desigual de poder, privilegio y acceso al bienestar que tienen hombres y mujeres. Es así como en la vejez el género explica la acumulación de desventajas y funciona como una categoría estratificadora que traduce la posioción económica para ambos sexos (CEPAL-CELADE, 2003).
Las criticas que se han hecho en este rubro iniciaron con romper la idea política del envejecimineto (hay que buscar soluciones prácticas) y el rostro femenino de la vejez, es decir, superar el problema social y dar espacio a la construcción de enfoques teóricos sobre género, envejecimineto y edad.

Relacionado con el género, en las últimas décadas, para conocer el bienestar de los mayores, se ha hecho énfasis en las formas de apoyo social, entre las que se destacan los apoyos familiares (Montes de Oca, 1999). Los apoyos familiares pueden ser considerados como tipos de ayuda que dan los miembros de una familia, independientemente que vivan o no con el anciano. En este tema se puede destacar que en la vejez los apoyos familiares toman una dimensión más importante que en otras edades, ya que a medida que avanza la edad, el ingreso por trabajo se reduce ante el cese de actividades generadoras de recursos económicos. En ese sentido, las transferencias monetarias son el indicador más adecuado para comprender el funcionamiento de los apoyos familiares y las redes sociales, que proporcionan recursos para satisfacer las necesidades de las personas envejecidas.

Un referente empírico de esta situación lo conforma un estudio comparativo basado en la encuesta sobre salud, bienestar y envejecimiento (SABE, 2001) de la Organización Panamericana de la Salud (OPS) realizado para siete ciudades en América Latina y el Caribe revela por ejemplo que en Buenos Aires el $59 \%$ de las personas mayores entrevistadas recibía ayuda en dinero; en São Paulo, este porcentaje alcanzaba al $61 \%$; en Bridgetown y Montevideo al 65\%, y en Santiago, La Habana y México, D.F., superaba el 70\% (Saad, 2003).

En este mismo tema pero en el plano local, una investigación demostró que una proporción significativa (alrededor del 30\%) de los hogares encabezados por personas mayores de 65 años, depende total o parcialmente de las transferencias informales de ingreso (Tuirán y Wong, 1993). Otro estudio que utilizó fuentes datos del año 2000 concluyó que una de las fuentes de ingreso más comunes de las personas mayores en el país era la ayuda familiar $(33,7 \%)$; los que dependían exclusivamente de la ayuda familiar alcanzaban casi al $10 \%$ de ellos (Wong y Espinoza, 2003).

Sin embargo, se ha advertido que no se pueden tomar los apoyos familiares como exclusivamente monetarios, ya que existen aquellos ligados al cuidado y acompañamiento. En este sentido, los ciudados hacia los adultos mayores forman parte de las transferencias entendidas como flujos de intercambio y circulación de recursos, acciones e información como lo sostienen Guzmán, Huenchuan y Montes de Oca (2003: 49). Esta dimensión de los apoyos sociales o familiares, cobra una relevancia mayor en países con deficientes sistemas de 
protección social, en donde los sistemas de apoyo informal (cuidados) ocupan un lugar preponderante en la calidad vida de los viejos.

Este tema es uno de los que necesitaba mayor evidencia empírica sobre los viejos y el entorno social de los mismos, sin embargo Robles, ha insistido mucho sobre el tema. Los cuidados aparecen como respuesta a la dependencia en la vejez que se define como "la condición social de un sujeto incapaz de satisfacer sus necesidades po sí mismo" (Robles, 2006: 145). Para la misma autora esta situación se explica de manera longitudinal, en cuanto: "Envejecer es un proceso de declive funcional, cognitivo, social, económico y político, el cual culmina con la muerte. La enfermedad crónica, por su parte, añade más dependencia a la vejez porque produce incapacidades de tipo biológico y funcionales progresivas e irreversibles, y finaliza también con la muerte" (Robles, 2006: 145).

Los cuidados sólo existen si hay dependientes, por lo tanto ambas situaciones aparecen juntas, con un nuevo actor social: el cuidador (Robles, 2006). Es de suma importancia mencionar que los cuidados se han centrado en el trabajo femenino más que en la familia u otros miembros de la comunidad. Más allá de los que se cree sobre la responsabilidad de la familia hacia los ancianos "casi siempre es una mujer quien asume dicha responsabilidad. La feminización del cuidado ha convertido una responsabilidad familiar y comunitaria en un asunto privado por que algunas mujeres de la familia se convierten en cuidadoras permanentes" (Robles, 2002 citado por Guzmán, Huenchuan y Montes de Oca, 2003: 55).

En definitiva, las investigaciones centradas en la familia y las redes sociales permiten dar cuenta de la importancia de los apoyos que de ellas se desprenden, como medio de compensación social ante la falta de mecanismos institucionales para satisfacer sus necesidades (Montes de Oca, 2001). Sin embargo, hay que tener en cuenta que aunque las familias funcionan de manera solidaria ayudando a sus viejos, esta situación se verá trastocada dada la sensible baja en las tasas de fecundidad. Aun así, no podemos prever el comportamiento de los núcleos familiares cada vez menos numerosas en el futuro, pero sí sabemos que se contará con menos miembros familiares disponibles para aportar recursos económicos y cuidados hacia los viejos. Finalmente, como menciona Montes de Oca (2001), en la literatura sobre los apoyos a los viejos existe mucha confusión e idealización romántica sobre el rol de la familia.

Otro rubro es la escolaridad, que es siempre un indicador relevante para medir el progreso y es también una la causa y consecuencia del desarrollo de un país, puesto que permite explicar cualquier otro fenómeno social o económico (Ham, 2003). Para el caso de México, las oportunidades de educación son diferentes en el tiempo y en el espacio situa- ción que se refleja en los rezagos actuales. Como consecuencia del avance en la cobertura educativa, son los jóvenes que cuentan hoy en día con mayores credenciales escolares; al interior de ellos muestran mejores indicadores los que se encuentran en las zonas urbanas en comparación con las rurales. Asimismo, el acceso a la educación ha sido más favorable para los hombres que las mujeres.

Se sabe que una buena parte de la población adulta mayor en México se encuentra en generaciones con en el llamado rezago educativo, dado que no ha completado la educación básica. Esta realidad limita las posibilidades de los ancianos en la sociedad y en la obtención de mejores condiciones de vida (INEGI, 2005). Por consiguiente, quienes enfrentan la vejez con un nivel educativo adecuado, tienen más herramientas para responder de manera activa y adaptarse mejor en la etapa de la vida adulta mayor.

México ha logrado importantes avances en cuanto a la educación con una franca ampliación de su cobertura, situación provocó una reducción de la tasa de analfabetismo que pasó del $25.8 \%$ en 1970 , al $9.5 \%$ en el año 2000 para el grupo de población de 15 años y más (INEGI, 1998 citado en INEGI, 2005).

A pesar de ello, los cambios en la educación no fueron homogéneos. En efecto, el 30\% de los adultos mayores no sabe ni leer ni escribir un recado, situación que afecta en un $50 \%$ más a las mujeres que a los hombres.

La tasa de alfabetismo permite comprender de manera más clara esta situación. El 35\% de las mujeres mayores de 60 años es analfabeta, contra 24\% por su parte a los hombres (Zúñiga y Vega, 2004). Este fenómeno no es extraño, dado que alrededor del $70 \%$ de los adultos mayores no ha finalizado el nivel primario. El resto del grupo se compone de proporciones muy bajas, en cuanto el $17 \%$ ha logrado terminar la primaria, un $1.1 \%$ corresponde a la secundaria completa, el 5.3\% ha logrado terminar la secundaria, y finalmente, $6.6 \%$ ha accedido a la educación media o superior (Zúñiga y Vega, 2004).

Dentro del mundo de los viejos, aparece una variable explicativa de su situación actual y que ha sucitado un gran interés; es el tema de la salud. Las investigaciones observadas señalan que la vejez se caracteriza por el aumento de los "riesgos de pérdidas en las capacidades físicas y mentales, disminución de la autonomía y la adaptabilidad, menoscabo de roles familiares y sociales, retiro del trabajo, pérdida de capacidad económica, cese de otras actividades y deterioros en la salud de consecuencias incurables y progresivas" (Ham, 1998: 32).

En este contexto resalta el hecho de que el envejecimiento de la población trae consigo una mayor demanda de servicios de salud, pero también demandas hacia la familia y la sociedad en gene- 
ral. El tema que más preocupa sin embargo es el desplazamiento de las causas en la morbilidad y la muerte de las enfermedades transmisibles hacia las crónicas, degenerativas e incapacitantes, que afectan principalmente en las edades más avanzadas (Frenk et al., 1990 citado por Ham, 1998).

Si bien este panorama es preocupante, dado que implica un fuerte aumento de los gastos en salud por parte de los gobiernos, aún más acuciante es la situación de los individuos que no cuentan con una correcta cobertura en salud. En este sentido, llegar a la vejez sin haber podido tener una cobertura médica básica, implica mayores riesgos o propensión a enfermedades no prevenidas, además de una obligación de conseguir recursos económicos para solventar los gastos en salud. En resumen, "Las enfermedades y las afecciones crónicas, así como el hecho de vivir solas, son factores de riesgo adicionales para las personas de edad" (ONU, 2010: 8).

En México este tema se relaciona con el término derechohabiencia, entendido como "las personas que están afiliadas a instituciones de seguridad social o tiene acceso a los servicios de salud" (INEGI, 2010: 4). Según el conteo de Población y Vivienda 2005, el $55.6 \%$ de los mayores de 60 años tienen una cobertura de salud (INEGI, 2010). Las evidencias son claras cuando mencionan que casi el $50 \%$ de la población envejecida no cuenta con cobertura en salud y aún mayor teniendo en cuenta el deterioro natural del cuerpo con el incremento de la edad.

En un nivel más práctico, cabe señalar que la falta de cobertura en salud puede ser el causante de estrategias económicas, sean éstas individuales o colectivas, para costear esa necesidad de atención. Para ello será necesario continuar con las investigaciones desde una mirada a lo largo de la vida y en especial en la vejez, el comportamiento de los adultos mayores en relación al mercado de trabajo, teniendo en cuenta también la salud como otros factores.

Una de las temáticas que ha desatado profundos debates académicos de todas las diciplinas y resultado en una reforma institucional, es el de los sistemas de pensiones, íntimamente relacionado con los conceptos anteriores. En las últimas décadas, este han atravesando problemas financieros, producto principalmente del aumento porcentual de los adultos mayores y como contrapartida se impusieron reformas. Frente a ello los gobiernos han optado en general por dos estrategias: una que toma el lugar de privatizar el sistema; en segundo lugar mantener un sistema con opción a público o privado, además de imprimir una extención del tiempo de cotización.

Aun así los resultados no han sido los mejores. En el telón de fondo de los debates y las reformas de los sistemas de pensiones deja entrever una mirada exclusivamente financiera a pesar de que los sistemas de pensiones se relacionan con aspectos demográficos, sociales y políticos.

El caso mexicano es analizado por Ham, Ramírez y Valencia (2008), quienes sostienen que la baja cobertura y monto, la desactualización, dispersión y la desigualdad son los principales conceptos que caracterizan los problemas que enfrenta el sistema de retiro en el país.

En cuanto a la cobertura, más del $60 \%$ de la población económicamente activa no cuenta con pensión y los mayores de 65 años que han obtenido el servicio es inferior al 20\% (Ham, Ramírez y Valencia, 2008). Existen muchos aspectos que deben mencionarse acerca de las pensiones en México, sobre todo porque es un tema inseparable de la calidad de vida de los mayores.

Ahora bien, no se puede dejar de mencionar un tema que ha sido objeto de un gran debate en los últimos años como lo es el tema de los derechos en la vejez. Es justamente a raíz de constatar las condiciones de vulnerabilidad en las que vive una gran parte de la población de la tercera edad, sus situaciones de pobreza, la falta de acceso a la salud, que ha impulsado a develar una situación alejada de los derechos de vivir una vida digna.

Revisar el derecho y las diferentes instancias de éste sobre los adultos mayores en el contexto mexicano, es de suma importancia. Principalmente interesa saber el marco legal a partir del cual el Estado controla, gestiona y resuelve la situación de un grupo de edad, en este caso la vejez, que según numerosas investigaciones (Huenchuan y Guzmán, 2003), se caracteriza por un alto porcentaje de vulnerables (aunque no todos comparten la misma situación).

Precisamente, ante la preocupación de la calidad de vida de los ancianos, se han formulado en el plano internacional y nacional numerosas leyes para acompañar el envejecimiento. El ejemplo más claro está en la Ley de los derechos de las personas adultas mayores publicada en el Diario Oficial de la Federación el 25 de junio de 2002.

Los diferentes artículos que la componen, hacen referencia a las garantías en vistas del derecho, que son inalienables para los adultos mayores. Por ejemplo, en el área laboral la ley menciona como derecho: "A gozar de igualdad de oportunidades en el acceso al trabajo o de otras opciones que les permitan un ingreso propio y desempeñarse en forma productiva tanto tiempo como lo deseen, así como a recibir protección de las disposiciones de la Ley Federal del Trabajo y de otros ordenamientos de carácter laboral" (Ley de los derechos de las personas adultas mayores, 2002).

Ahora bien, no hay norma jurídica que pueda separarse de un valor, ni de la costumbre o la moral que da orígen al derecho. Parece que en México, más alla de la ley, la costumbre y la cultura, son las 
guías de comportamiento más importantes y que se reparten con la ley, el monopolio legítimo del control social. Es la misma ley por tanto, que da sentido a la vulnerabilidad de las personas de la tercera edad, quiénes presentan en su mayoría problemas en el acceso al bienestar y la protección social.

En 2006, la Cepal impulsa una agenda para lograr el consenso en América Latina y el Caribe sobre la proteción, en donde los adultos mayores fueron una parte importante de dicho consenso (Huenchuan, 2009). Siguiendo con el mismo texto, la autora pregona por una responsabilidad departe de los Estados para "promover el pleno goce de los derechos de las personas mayores, creando las condiciones jurídicas, políticas, económicas, sociales y culturales que permitan el desarrollo íntegro de la persona humana" (Huenchuan, 2009: 33).

Este reclamo es coherente con el vacío que se detecta entre la cobertura legal y la cobertura real de los derechos económicos, sociales y culturales para los adultos mayores y en general para el conjunto de la sociedad (Huenchuan y Morlachetti, 2007).

Otro punto relevante con respecto a los derechos en la vejez, es que detrás de este tema se encuentra el desafío de una sociedad más justa, inclusiva y solidaria, no solamente para los viejos, sino para todas las edades.

Por último, encontramos las actividades laborales en la vejez, tema que ha sido investigado y que en el caso de México, en comparación con otros países, llama la atención que en las edades avanzadas siguen participando económicamente.

No obstante, la actividad laboral de los adultos mayores es un fenómeno que se manifiesta en todo el mundo, pero con dinámicas e intensidades diferentes según la región o el país que se estudie; lo que sí es claro es que se esta frente a un fenómeno que abarca a una amplia proporción de éstos sujetos. Por ejemplo, en 2008 a nivel global, los mayores de 65 años de edad económicamente activos representaron el $30 \%$ de los hombres y $12 \%$ de las mujeres (ONU, 2010).

En el caso de México, un trabajo realizado en base al XI Censo de Población y Vivienda de 1990, el INEGI (1990) muestra que en el transcurso de 1970 al año 1990 la tasa de participación de los adultos mayores se redujo en un $12.8 \%$, dado que este indicador pasó de $41.4 \%$ a $28.6 \%$ para ambos sexos (INEGI, 1990). A pesar de ello, no se puede hablar de una tendencia clara hacia la reducción, porque desde 1990 hasta nuestros días, se constata contrariamente un aumento del mismo indicador.

Teniendo en cuenta los datos que arroja el XII Censo del 2000, éstos relevan que el $55.1 \%$ del grupo de edades de 65 a 69 años aún permanece activo, $45 \%$ para el grupo de $70-74$ y finalmente la proporción de mayores de 75 años y más que trabaja alcanza al 28.7\% (Ham, 2003).
Sin embargo, es lógico que a medida que avanza la edad la posibilidad de trabajar es menor; si bien puede atribuirse esta idea al natural deterioro físico y mental del cuerpo, habría que agregar otras variables como el género y los apoyos familiares que son también influyentes. Lo cierto es que conforme avanza la edad hay una mayor dificultad para permanecer en el mercado de trabajo, hasta llegar a la ausencia total de actividad. No obstante, a los 80 años, uno de diez varones mexicanos sigue trabajando (Zúñiga y Vega, 2004).

Esta es una evidencia irrefutable de que en México no existe para la mayoría de la población, un límite de edad, en los hechos, para trabajar. Basta con observar la vida cotidiana del país para corroborar lo dicho. Los supermercados mexicanos son un buen ejemplo de la ausencia de límite de edad para trabajar, dado que los conocidos "paqueteros", son mayores de 65 años de edad y otros menores de 12 años. Esto es congruente con un mercado de trabajo que es sumamente heterogéneo, en el sentido que las formas laborales son diversas (y se han diversificado aún más en las últimas décadas).

En realidad no se puede decir que el trabajo en la vejez sea por sí mismo un problema social, después de todo esto ha siempre sucedido. Sin embargo, la relación entre la economía y el envejecimiento, el mercado y la vejez y aún más importante, la edad y el trabajo son hechos sociales que son necesarios poner en relación para comprender un aspecto fundamental de la vida de los viejos en México.

Esta situación adquiere rasgos propios al paisaje económico mexicano, que ha sido el objeto de hondas transformaciones económicas (desregulación laboral, privatizaciones, ajustes económicos) que han repercutido en el mercado laboral y en consecuencia en la vida laboral de las personas.

No obstante, al problema actual se le suma una preocupación mayor considerando que las tendencias demográficas presentan escenarios en donde los viejos ocuparán, en los próximos 40 años, un peso porcentual y absoluto mayor que en la actualidad y esto a nivel laboral genera interrogantes acerca de cómo responder a nuevas demandas, con un mercado laboral que ya se caracteriza por funcionar con una mayoría de trabajadores en el sector informal, sin protección social y que llevan a situaciones de pobreza extrema al llegar la vejez.

\section{La construcción social de la vejez}

Los temas que se han destacado corresponden como se mencionó, a una selección del desarrollo histórico de investigaciones y autores que lograron instalar el debate sobre la vejez y las características en México. Pero este trabajo quedaría incompleto sin afrontar la difícil tarea de develar la construcción social de la vejez. 
Para comenzar, es menester señalar que la aproximación a la vejez no se puede entender acabadamente en el vacío sino, en un contexto sociohistórico particular que le da sentido. La imagen que se construye sobre la vejez es producto histórico-cultural que refleja imaginarios diferentes que son construidos desde la sociedad y las instituciones, como es por ejemplo la imagen de la vejez como un momento de descanso después de años de aporte laboral o, desde otro lado, la imagen de la vejez como dependencia y pobreza. Estas caras de la vejez son ante todo el resultado de la construcción social a partir de la edad.

Estos heterogéneos imaginarios alrededor de la edad como categoría social indican que la vejez hay que abordarla como una construcción social. En este sentido se coincide con Kehl y Fernández (2001), quienes sostienen que hay una careta o disfraz detrás de la vejez que circula socialmente. A pesar de ello es posible desenmascarar algunas cuestiones que participan en la construcción de las etiquetas, el problema reside en su característica principal es que se incorporan socialmente por mecanismos muy sutiles. Por ejemplo, hoy en día enunciar la palabra Viejo en México tiene una carga negativa en cuanto nos envía a una situación poco feliz o deseada de muchos de los seres humanos.

Las casusas se encuentran en que el concepto se ha amalgamado con un estereotipo negativo imágenes - , que no necesariamente coincide con características de vida de todos nuestros ancianos. Esto también tiene que ver con la lucha por el posicionamiento de las edades en la escala social, donde la juventud es identificada como la belleza y vigor, contra la vejez con la imagen de lentitud, deterioro, debilidad y dependencia.

De esta manera, bajo el ethos de la modernidad, el éxito, la competitividad, velocidad y agresividad, son claramente identificados con la juventud y las personas de la tercera edad son presentadas como anacrónicas (Rodríguez, 1979). Esta visión no distingue de clase social, raza, o color de piel; retiro e inutilidad, quedan entrelazados a la imagen de la vejez, y trasforma los prejuicios, en hechos casi irrefutables.

Hay que recordar que las clasificaciones y categorías del mundo social, hasta las más naturales y objetivas, nos comunican a los fundamentos sociales. La edad, como propiedad biológica, sirve de criterio de clasificación de los individuos en el espacio social. Por lo tanto, las etiquetas sociales no son naturales, sino socialmente construidas.

Finalmente la edad es una noción social, establecida en comparación con otros grupos. En esa comparación, se pone en juego el poder de los diferentes ciclos de vida ${ }^{1}$. Esta variable es un buen espacio para analizar las relaciones sociales entre generaciones, clases sociales y las representaciones dominantes de las prácticas legítimas asociadas a la definición de la edad.

\section{Reflexiones finales: las deudas en la investigación sobre la vejez en México}

Una recapitulación del análisis social del envejecimiento y la vejez en México muestra que se han producido avances en muchos temas - aunque todavía estas temáticas no han sido agotadas entre ellos destacan los aspectos demográficos, las pensiones, la atención y el cuidado con los apoyos familiares, la escolaridad, la salud, la vulnerabilidad, los derechos en la vejez y la participación económica de las personas mayores. Sin embargo, no todos los temas han recibido la misma atención a pesar de conocerse su existencia.

Después de una revisión sobre las temáticas más relevantes que tratan sobre la situación de los viejos, se puede subrayar que en la mayoría de ellas existe un acuerdo en que una de las principales preocupaciones del envejecimiento en México es el rezago social en que se encuentra este grupo y la urgencia en la cual se deben imprimir mejoras en sus situaciones. Las características de la sociedad mexicana son poco favorables para el panorama del envejecimiento demográfico, ya que existe una correlación entre ancianidad y pobreza como resultado de la falta de ahorro o acumulación de bienes durante la trayectoria de vida de una persona (Ribeiro y Mancinas, 2009). Aunado a esto, el envejecimiento demográfico está impulsando cambios en los individuos y en la sociedad al trastocar los deberes y los roles con respecto a la edad.

Dadas las condiciones económicas y sociales actuales en México, además de los cambios que han sucedido a lo largo de las últimas décadas (transformación del modelo económico-político), que han subvertido los escenarios y personajes, esto impulsa a la imaginación científica a pensar sobre nuevas respuestas al comportamiento de la población mayor.

Porque como menciona una autora "Los cambios sociales obligan a pensar en una nueva vejez; a introducir nuevos análisis en diversos niveles, e incluir otros fenómenos atravesados por el envejecimiento. El estudio de la vejez lejos de estar agotado, está abierto a múltiples posibilidades analíticas" (Robles, 2006: 142).

Hay que recordar que las diferentes temáticas tratadas, algunas se han presentado aquí, conforman un zócalo desde el cual debemos lanzar nuevos interrogantes sobre el comportamiento de la población de la tercera edad. El desafío no es menor, teniendo en cuenta que la acumulación de la cantidad de sujetos mayores de 60 años está en permanente aumento. 
Una nueva agenda de investigación necesita superar el debate entre lo exclusivamente teórico y lo práctico. Se necesitan realizar aportes tanto teóricos como prácticos, en el tema de la acción social de que llevan los sujetos a cabo en diferentes contextos sociales. En términos teóricos esto puede abordarse desde el enfoque de las estrategias que también permite incorporarse con el debate que sostiene la imposibilidad de los sujetos de ser conscientes de la dirección de sus trayectorias de vida, frente a las teorías que por el contrario, insisten en la incapacidad racional de los sujetos en torcer el destino de los caminos sociales predeterminados.

En el área de la práctica, el aporte se puede extender a las políticas sociales y en particular aqueIlas que tienen como beneficiarios a las personas de la tercera edad. Lo que estas personas viven cotidianamente, sus redes y estrategias, son fundamentales de conocer para adecuar la intervención social y mejorar su calidad de vida.

Resaltando la aportación práctica, pero sin olvidar la relación con la teoría, una nueva agenda de investigación necesita desentrañar un fenómeno que es complejo y comprenderlo a partir de los actores mismos. Se sostiene que los fenómenos sociales planteados como causas-efecto se pueden enfrentar a graves errores si no se tiene en cuenta la dimensión temporal. Así, las crisis económicas pueden desatar efectos en el corto plazo que "pueden ser confundidos con tendencias de largo plazo en la estructuración de los mercados de trabajo" (Solís y Billari, 2003: 561).

Como se mencionó inicialmente, México cuenta con pocos años para adaptar las estructuras institucionales que se verán afectadas por la presencia de un mayor porcentaje de ancianos. Ciertamente, si lo que se busca es un futuro con mejor bienestar para esta población y sus entornos. Lo que nos lleva a que el camino para logar un régimen de bienestar digno "comienza por generar conocimiento de las condiciones, oportunidades y problemas que enfrenta la población que envejece, con objeto de identificar estrategias y posibilidades" (Ham, 2003).

\section{Notas}

1 También conocido como curso de vida, esta perspectiva ha sido inicialmente trabajada desde la Psicología, pero desde la Sociología se ocupa principalmente por analizar procesos y, entre otras cosas, las trayectorias de vida de los individuos y sus interrelaciones (Blanco, 2011).

\section{Referencias bibliográficas}

ACEVEDO, J.; F. Bruno; M. Trujillo y M. Lopez (2015), "La calidad de vida de los adultos mayores, los grandes desafíos", en Problemática de los grupos vulnerables visiones de la realidad, Tomo VII, UAdeC, FTS.
ARANIBAR, P. (2001), "Acercamiento conceptual a la situación del adulto mayor en América Latina", Serie Población y Desarrollo, no 21, CEPAL-FNUAP.

ARBER, S. y J. Ginn (1996), Relación entre género y envejecimiento. Enfoque sociológico, Madrid, Narcea Ediciones.

BLANCO, M. (2011), "El enfoque del curso de vida: orígenes y desarrollo", Revista Latinoamericana de Población, 5 (8), pp. 5-31.

BUENO, A. y A. Dávila (2010), "Textos y contextos del envejecimiento en México", en S. M. Ribeiro, La población de tercera edad en Mexico: políticas de atención, perspectivas y retos, México, Plaza y Valdes, pp. 135-148.

CEPAL-CELADE (2003), "Las personas mayores en América Latina y el Caribe: disgnóstico sobre la situación y las políticas", en Conferencia regional intergubernamental sobre envejecimiento, Santiago de Chile, CEPAL.

CONAPO (2003), Índices de desarrollo social en las etapas del curso de vida, 2000, México, Consejo Nacional de Población.

GUTIÉRREZ, J.; A. Serralde y M. Guevara, (2007), "Prevalencia de desnutrición del adulto mayor al ingreso hospitalario", Nutrición Hospitalaria, no 22, pp. 702-709.

GUZMÁN, J.; S. Huenchuan y V. Montes de Oca (2003), "Redes de apoyo social de las personas mayores: marco conceptual", Notas de Población, 30 (77), pp. 35-70.

HAM, R. (1998), "Implicaciones del envejecimiento en la planeación del bienestar", Papeles de Población, CEPAL, 4 (17), pp. 31-38.

HAM, R. (2003), El envejecimiento en México: el siguiente reto de la transición demográfica, México, Miguel Ángel Porrua.

HAM, R.; B. Ramírez y A. Valencia (2008), Evaluación y tendencias de los sistemas de pensiones en México, México, Miguel Ángel Porrúa.

HUENCHUAN, S. (2009), Envejecimiento, derechos humanos y políticas públicas, Santiago de Chile, Naciones Unidas.

HUENCHUAN, S. (2011), Los derechos de las personas adultas mayores. Materiales de estudio y divulgación, Santiago de Chile, CELADE - CEPAL.

HUENCHUAN, S. y A. Morlachetti (2007), Derechos sociales y envejecimiento: modalidades y perspectivas de respeto y garantía en América Latina, Santiago de Chile, CEPAL.

HUENCHUAN, S. y M. Guzmán (2003), "Seguridad económica y pobreza en la vejez: tensiones, expresiones y desafíos para el diseño de políticas", Notas de Población, no 83, pp. 99-125.

INEGI (1990), La tercera edad en México, 1993, IX Censo General de Población y Vivienda, México, INEGI.

INEGI (2005), Los adultos mayores en México. Perfil sociodemográfico al inicio del siglo XXI, Aguascalientes, INEGI. 
INEGI (2010), Principales resultados del censo de población y vivienda 2010, México, INEGI.

JOHNSON, M. (2001), "La conception de la vieillesse dans les théories gérontologiques", Retraite et Société, 3 (34), pp. 51-67.

KEHL, S. y J. Fernández (2001), "La construcción social de la vejez", Cuadernos de Trabajo Social, no 14, pp. $125-161$.

LEY DE LOS DERECHOS DE LAS PERSONAS ADULTAS MAIORES (2002), México, Diario Oficial de la Federación el 25 de junio de 2002.

MONTES DE OCA, V. (1999), "¿Envejecimiento? Una discución sobre la edad y su relación con el empleo, retiro y reproducción social", Taller sobre envejecimiento, empleo y previción social, Tepoztlán.

MONTES DE OCA, V. (2001), "Bienestar, familia y apoyos sociales entre la población anciana en México: una relación en proceso de definición", en C. Gomes (org.), Procesos sociales, poblacion y familia. Alternativas teóricas y empíricas en las investigaciones sobre la vida doméstica, México, Facultad Latinoamericana de Ciencias Sociales y Migual Ángel Porrúa, pp. 345-376.

MONTES DE OCA, V. (2010), "El adultos mayor en situación de pobreza y vulnerabilidad en el área metropolitana de Monterrey: retos para la política pública", en M. Ribeiro y S. Mancinas (orgs.), Textos y contextos del envejecimiento en México, México, Plaza y Valdés, pp. 15-40.

OMS (2015), Envejecimiento y salud, OMS.

ONU (2010), Seguimiento de la Segunda Asamblea Mundial sobre el envejecimiento: análisis exhaustivo, $A / 65 / 157$.

OSORIO, P. (2006), "La longevidad: más allá de la biología. Aspectos socioculturales", Papeles del CEIC, 6 (22), pp. 1-28.

PACHECO, E. (2009), "Las políticas de población en México. Debates y propuestas para el Programa Nacional de Población 2008-2012", Memorias del Foro Nacional, México, CONAPO, pp. 177-178.

PARRA, C. y A. Quintero (2007), "Mínimo vital y los derechos de los adultos mayores", Estudios Socio-Jurídicos, vol. 9, pp. 236-261.

PARTIDA, V. (2005), Proyección de indígenas de México $y$ de las entidades federativas 2000-2010, México, CONAPO.

REYES, M. (2006), Análisis demográfico de la población de adultos mayores en México, 2006-2050, México, Cámara de Diputados.

RIBEIRO, M. y S. Mancinas (2009), Textos y contextos del envejecimiento en México, México, PLaza Valdés.

ROBLES, L. (2006), "La vejez: nuevos actores, relaciones sociales y demandas políticas", Relaciones, XXVII (105), pp. 140-175.
RODRÍGUEZ, C. (1999), "El papel de las instituciones públicas en la atención de la tercera edad", en CONAPO, El envejecimiento demográfico de México: retos y perspectivas, México, CONAPO, pp. 55-68.

RODRÍGUEZ, J. (1979), "Perspectiva sociológica de la vejez", ReiS, n.० 7, pp. 77-97.

SAAD, P. M. (2003), "Transferencias informales de apoyo de los adultos mayores en América latina y el Caribe; estudio comparativo de encuesta SABE", Notas de Población, n. ${ }^{0}$ 77, pp. 175-217.

SABE (2001), Encuesta sobre salud, bienestar y envejecimiento, Kingston, Organización Panamericana de la Salud.

SÁNCHEZ, D. (2008), "Adultos mayores en la planeación del espacio turístico rural en Tamaulipas", Papeles de Población, 14 (55), pp. 59-94

SÁNCHEZ, P. (2000), "Sociología de la vejez versus economía de la vejez", Papers 61, pp. 39-88.

SCOLICH, N. (2005), "Pensar la vejez", Cartapacio de Deracho, vol. 9, pp. 1-55.

SEDESOL (2010), Primer informe trimestral 2010, México, Gobierno Federal.

SERRANI, D. (2012), Distinción entre envejecimiento y vejez, Buenos Aires, Argentina.

SOLÍS, P. y F. Billari (2003), "Vidas laborales entre la continuidad y el cambio social: trayectorias ocupacionales masculinas en Monterrey, México", Estudios Demográficos y Urbanos, n. ${ }^{\circ}$ 54, pp. 559-595.

TUIRÁN, R. (1999), "Desafíos del envejecimiento demográfico en México", en CONAPO, Envejecimiento demográfico en México. Retos y perspectivas, México, CONAPO, pp. 16-22.

TUIRÁN, R. y Wong, R. (1993), "Transferencias familiares en el envejecimiento", en Seminario sobre envejecimiento demográfico en México, México, Somede, Mimeo.

VIVEROS, A. (2001), "Propuestas para la formulación de políticas públicas y fomento de acciones desde la sociedad civil en América Latina y el Caribe", CEPAL - Series de Población y Desarrollo, n. ${ }^{\circ}$ 22, pp. 64.

WONG, R. y M. Espinoza (2003), "Ingresos y bienes de la población de edad media y avanzada en México", Papeles de Población, n.o 37, pp. 129-166.

YUNI, J. A. y C. A. Urbano (2008), "Envejecimiento y género: perspectivas teóricas y aproximaciones al envejecimiento femenino", Revista Argentina de Sociología, 6 (10), pp. 151-169.

ZÚÑIGA, E. y C. Gomes (2002), "Pobreza, curso de vida y envejecimiento poblacional en México", en CONAPO, La situación demográfica de México 2002, México, CONAPO, pp. 141-153.

ZÚÑIGA, E. y D. Vega (2004), Envejecimiento de la población de México. Retos del siglo XXI, México, CONAPO. 
Recebido a 23/11/2015. Aceite para publicação a 23/11/2016.

Fernando Bruno (fernandobruno77@hotmail.com). Universidad Autónoma de Coahuila, Facultad de Trabajo Social, Cuerpo Académico de Grupos Vulnerables. Avenida Dr. ${ }^{a}$ Cuquita Cepeda de Dávila, s/n, Col. Adolfo López Mateos, 25125 Saltillo, Coahuila, México.

Jesús Acevedo Alemán (jesusaceve@hotmail.com). Universidad Autónoma de Coahuila, Facultad de Trabajo social, Cuerpo Académico de Grupos Vulnerables. Avenida Dr. a Cuquita Cepeda de Dávila, s/n, Col. Adolfo López Mateos, 25125 Saltillo, Coahuila, México. 